\title{
Putting More Brain-Like Intelligence into the Electric Power Grid: What We Need and How to Do It
}

\author{
Paul J. Werbos \\ National Science Foundation ${ }^{1}$ \\ Arlington, Virginia, US \\ werbos@ieee.org
}

\begin{abstract}
Certain key features of brain-like intelligence are essential to fulfill the main goals of policy-makers and environmentalists for the "smart grid" - a key item in the new economic stimulus law, and a key item in a rational strategy for energy sustainability. This paper will explain why and how, and how the neural network community could play a crucial role in making this real.
\end{abstract}

\section{THE GLOBAL NEED}

In any engineering application, it is necessary to start out by spending some time in problem formulation, in order to avoid wasting time and effort in well-crafted useless sophisticated dodo birds, which have become all too common in recent years. "Smart grid" technology is a prime example of this. At NSF, a careful evaluation of "broader benefits" is a major part of deciding which research is worth funding.

Many people (for marketing reasons) use the term "smart grid" to mean conventional types of investment in building wires and substations, in order to reduce the chances of future blackouts. There is a need for such investments, but they do not require computational intelligence. While useful, they are not enough by themselves to meet some of the very urgent needs of the world energy economy. Some of the short-term investment needs could be met more efficiently by importing technologies like the SIL wiring technology from Brazil, which often allows $60 \%$ more electricity to be carried on a given right-of-way, and could have been very useful to the state of California in reducing its budget problems after the electricity shortages a few years ago[1].
A new vision for an intelligent grid was first crystallized in two back-to-back workshops in Mexico, planned by James Momoh, Massoud Amin and myself, jointly led by NSF and the Electric Power Research Institute (EPRI) [2].

The practical and urgent need for this new vision has become clear in recent years. Many people now believe that new energy sources like wind offer our only possible escape from a combination of terrifying threats from greenhouse gasses, nuclear proliferation resulting from a flow of enrichment technologies to every part of the earth, and conflicts due to growing scarcity of energy resources. [3]. But new, marginal sources of wind power cost 20 cents per kwh in Europe - about three times what traditional sources cost - and are not available when electricity is needed most. If had enough energy storage in the power grid, and if we had the right kind of intelligent control, existing wind farms could give us three times as much electricity as we now get from them, at the times when we want the electricity; when we get three times the electricity from the same capital investment, it cuts the cost per kwh by a factor of three, and suddenly makes it affordable to make a massive conversion of the world's energy system starting now.

I would like to give special thanks to Georg Zimmerman of Siemens and the IEEE CIS Alternate Energy Task Force, and to the IEEE-USA Energy Policy Committee, for explaining some of the practical ramifications here to me. There are only three renewable energy sources - wind, solar farms and energy from space - which clearly could supply all of the energy needs of the entire US. Of these three,

${ }^{1}$ During 2009, the author is assigned off-site to legislative activities not representing NSF. This work is in the "government public domain;" it may be copied without restriction, so long as it is properly cited. It does not represent the views of anyone but the author. 
wind is the only one mature enough to be ready for deployment today on that scale. Even as we build new wind farms, and build the wires to connect the grid to regions of strong wind in the Rocky mountains and offshore, we need to work equally hard on the storage and on the specific kind of intelligent control that we need in order to make full use of that storage and of other ways to shift the timing of electricity supply. This need is now widely recognized among policy makers; it is a major reason why the "intelligent grid" has been allocated on the order of a billion dollars in the economic stimulus bill which passed this month (February 2009 [4]). Largescale storage and intelligent control would still be necessary in meeting total US needs even if we also developed solar farms at a cost of 6 cents per kwh - a reasonable goal well beyond the constrained R\&D efforts now underway.

The storage part of this challenge is also of great importance - not just for wind power, but for the changes we need to make in cars and trucks [5]. However, there are a number of very promising efforts to develop and deploy advanced technologies to address that part; for example, see [6], see the practical efforts to commercialize compressed air storage technology, and see discussions at Congress, DOE, NSF and DOD about options for new efforts. The ability to make full (optimal) use of this storage, and of other methods for timeshifting electricity supply and demand, is the critical barrier here - the barrier which computational intelligence may be necessary to overcome.

Strictly speaking, computational intelligence is also important on the storage side. Intelligent battery management systems, and intelligent exploration of the design space, can play an essential role there [6]. Intelligent enduse systems, such as the reinforcement learning control for Heating, Ventilation and Air Conditioning by Hittle, Anderson and Young, could be extended to respond to price fluctuations and give more "control authority" to an intelligent grid. Price-responsive management of distributed storage is described in my patent assigned to Greensmith [6] - but we can only get the full value from such systems if the right price signals are broadcast from the grid itself. Other authors such as Harley, Venyagamoorthy, Monoh have reviewed a variety of other ways that computational intelligence can be used to improve other key components of the electric power grid. Harley and Venayagamoorthy, especially, have begun to scale up to experiments on Wide Area Control (WAC) [7] which come closer to a true intelligent grid than anything else implemented to date.

The remainder of this paper will discuss the technical details of the intelligent grid challenge itself, and the connection to neural networks.

\section{THE TECHNOLOGY TO MEET THE NEED}

In a sense, the challenge here is simply to build a better controller for the electric power grid. Many people say that the electric power grid is the most complex single piece of machinery ever built by humans. Even so, we can view this as nothing but a testbed for the general problem of how to design an adaptive, optimal controller for a complex nonlinear system subject to random disturbances.

That is exactly the focus of adaptive approximate dynamic programming (ADP) [2,8], a major emerging thrust of computational intelligence. But in order to handle general nonlinear decision problems, the best ADP designs all require several components which learn to approximate unknown nonlinear mappings. Neural networks are essential to handling this task well, in general complex environments, because several types of neural networks provide more accurate universal approximation than any classical alternatives $[9,10]$. They also make it possible to use new chips in the Cellular Neural Network family which already offer thousands of processors in parallel on a single commercially available chip.

How does this mathematics map into the practical realities of trying to upgrade the electric power grid? How and why can we apply ADP directly to the global problem of controlling the entire electric power grid?

To begin with, we need to review some key aspects of how the electric power grid is controlled today $[2,11]$. There are many, many control methods used for individual components of the grid - but these methods usually do not control all the pieces together to make them optimize the overall performance of the grid as a whole. For control of the grid as a whole, the best tool available today is Optimal Power Flow (OPF). James Momoh of Howard University has been the leader in developing an integrated suite of optimization tools which make it possible to coordinate a wide collection of control decisions 
across the entire grid, to get optimal performance of the system as a whole. OPF and ADP are the two foundations which we proposed to integrate and build upon, starting in 2002.

What are the limits of today's OPF, and why do we need something better? OPF calculates the best possible combination of control actions across the grid, in order to maximize some measure of performance $U(\underline{\mathbf{x}})$, based on the best available models of how the grid works and assuming no significant random disturbances. (Evolutionary computing is part of that suite.) As part of that calculation, it calculates a vector $\underline{\lambda}$ of "shadow prices" for all of the state variables in the system. In recent years, economists and power engineers have worked together to develop new ways to manage and regulate electric power grids based on a new concept of "locational marginal cost," which is essentially just a new way of package this vector $\underline{\lambda}$ calculated by OPF! Strictly speaking, the market is solving a multicriterion optimization problem here, but it still ends up with the same mathematics and the same $\underline{\lambda}$ vector!

Great and useful as all of this is, it does not do anything at all to tell us how to shift power or storage from one time to another. It only maximizes a measure of present benefits from present actions. These locational marginal costs do not account for the value of storing power instead of selling it immediately.

There have been a variety of quick fixes used to address such problems, beyond the scope of today's OPF. For example, in managing large pumped hydro storage of electricity, Chile has made widespread use of Dynamic Programming (DP) proper. DP proper does address the crosstime optimization problem and it does allow for random disturbances - but no one can afford the computational cost of applying DP proper to more than a handful of variables at a time. The cost of this approach is that one can only account for a handful of state variables in doing the optimization; the approximation is extreme, and tolerable only when nothing better is available. $\mathrm{L}$ As another example, people have used complex auctions of human actors in order to plan unit commitments and generation, hour by hour, a day to years in advance. But when generation schedules are set up years in advance, it is hard to make use of the power one gets when the wind suddenly and unpredictably starts blowing hard in the middle of the night. There are certain other problems as well in the gaming of such systems, as exhibited by Enron and others. Some have even proposed that human consumers should be asked to watch price signals at 15 minute intervals, every hour of the day, and in the middle of the night; it may be more practical and efficient to learn how to delegate more of these tasks to computer systems based on computational intelligence, guided by humans in more human-friendly ways.

As an alternative - we can use adaptive critic networks to provide the necessary price signals.

In reality, the intelligent grid of the future would not be one integrated physical computer system directly controlling every battery and switch in the United States. For example, each individual house might have its own intelligent control system, with basic values tuned by the user, and with foresight and adaptability of its own. In order to function efficiently as part of a larger power grid (power market), it needs to be responsive to real-time electricity price signals coming to it from the grid. For example, when the wind blows, the larger grid somehow needs to tell the household "now would be a good time to charge up your battery"; instead of controlling the customer's house in detail, it can simply send a price signal which says "I'll sell you the electricity much cheaper for the next 15 minutes.” Many parts of the US already broadcast real-time electricity prices every 5 or 15 minutes - but we need better technology to generate more accurate price signals, and to make better use of the price signals.

Where do these real-time price signals come from? Again, locational marginal price does not give the correct market signal for the cross-time optimization problem, no matter how it is jiggered up. Mathematically, the correct vector of shadow prices $\underline{\lambda}$ for the case of crosstime optimization with noise is identical to the vector of “value signals” approximated by Dual Heuristic Programming (DHP), the most powerful of the popular ADP methods. (For example, at the IEEE ADPRL07 conference, Warren Powell showed how he improved the performance of some of his classical, demanding work in ADP by applying what amounts to ADP for the nonlinear function forms he has designed for his specific class of applications in largescale logistics coordination.) We can also calculate $\underline{\lambda}$ as the gradient of the output of a more conventional scalar critic; it is an easy calculation using backpropagation.

To take the most straightforward path how can we train a neural network DHP critic to evaluate the state of the entire electric power 
grid? The traditional methods for training critics for a handful of turbogenerators and switching devices would simply not work here, because of the sheer size and complexity of the electric power grid.

In fact, this is a beautiful example of convergent evolution between biology and evolution. Biology, too, has had to address survival in environments far more complex than the 10-50 sensor input variables we use in most engineering control problems today. Even reptiles and amphibians certainly learn how to learn nonlinear relations in visual fields which comprise many thousands of variables. Engineering is not yet ready to start implementing the full range of capabilities which biology uses to handle complexity [8], but to handle the electric power grid as a whole effectively, we do need to implement a few of the next steps which move us in that direction. Multilayer perceptrons already handle complexity far better than linear basis function designs like Taylor series or local interpolators like lookup tables, radial basis functions, and networks trained by Hebbian learning [9]. But to handle the power grid, we need to move beyond multilayer perceptrons to networks which are more difficult to train but more powerful in handling complexity.

There is already an entire family of such methods in existence today, ranging up from the cellular SRN [10] to the full-fledged recurrent ObjectNet. The cellular SRN is very convenient for input fields distributed across a Euclidean grid, like the pixels of an image. It is also a good place to start in building up software and early applications. However, the topology of the electric power grid (in a proper compact description) is not Euclidean; it is more like a fishnet of objects and connections. The fullfledged recurrent ObjectNet is necessary, in order to generalize the CSRN capabilities to that kind of non-Euclidean case. Instead of mapping a complex input field into $\mathrm{M}$ rectangular cells, all governed by a common “inner loop” neural network, one may map it into a network of $\mathrm{k}$ types of “Objects," with k different types of “inner loop” neural networks. A brief but complete formal specification is given in IJCNN tutorials posted in the neural network subheading at www.werbos.com.

A patent has been filed via the University of Memphis for the training of CSRN, of Object Nets, and of Cellular Neural Network structures (all of which are closely related) as described in [10].
The feedforward version of ObjectNets has been implemented so far by two groups - by Harley and Venayagamoorthy's group in widearea control of the grid [9], and by David Fogel's group in training a computer to play chess [12]. Both used evolutionary computing to train the critic network. Fogel's system was the world's first computer system to achieve master-class performance in chess without using a supercomputer and without using detailed clues and advice from a human; it learned how to play the game at that level. This is already a major milestone in replicating brain-like intelligence, but the true intelligent grid based on recurrent ObjectNets - centralized or distributed in its physical implementation - would be major step even closer, of enormous importance to the future of humanity.

\section{REFERENCES}

[1] Chen-Ching Liu, report of the joint workshop of NSF and the Electric Power Research Institute (EPRI), held at EPRI headquarters (Palo Alto, California), October 2001.

[2] See the chapters by Momoh and myself in J. Si et al (eds) Handbook of Learning \& Approximate Dynamic Programming Wiley/IEEE 2004. Also see the workshop web site www.eas.asu.edu/ nsfadp and "Toward a Smart Grid," (Amin and Wollenberg), IEEE Power and Energy Magazine, Vol.3, No 5, pp. 34-38, Sept/Oct. 2005

[3] P. Werbos, Technological Solutions for Energy Security and Sustainability. In Gal Luft and Anne Korin, eds., Energy Security Challenges for the $21^{\text {st }}$ Century: A Reference Handbook (C9997), Greenwood: Westport, CT, 2009. For more details, see www.setamericafree,org and http://precedings.nature.com/documents/2131/ version/1

[4] See the text of HR1 of the $111^{\text {th }}$ Congress, as passed jointly by the House and Senate, posted at http://thomas.loc.gov

[5]See [3], and www.werbos.com/E/500mpg.pdf. [6] See [5], http://greensmith.us.com/ and //web.mit.edu/dsadoway/www/nsfworkshopMain.htm J.Von Neumann and O.Morgenstern, The Theory of Games and Economic Behavior, Princeton NJ:

Princeton U. Press, 1953.

[7] Wei Qiao, Ganesh Venayagamoorthy and Ronald Harley, DHP-Based Wide-Area Coordinating Control of a Power System with a Large Wind Farm and Multiple FACTS Devices, In Proc. of the International Joint Conference on Neural Networks (IJCNN07). IEEE.

[8] P. Werbos, Intelligence in the Brain: A Theory of How it Works and How to Build It. Accepted 
for special issue of Neural Networks, edited by Kozma and Levine, forthcoming 2009.

[9] A.R.Barron, Universal approximation bounds for superpositions of a sigmoidal function, IEEE

Trans. Info. Theory 39(3) 930-945, 1993.

[10] Ilin, R., Kozma, R. \& Werbos, P. J, Beyond Feedforward Models Trained by Back-

propagation: A Practical Training Tool for a More Efficient Universal Approximator, IEEE

Transactions on Neural Networks. Vol. 19, No.6, June 2008. p. 929-937. Also see [8].

[11] James Momoh, Electric Power System Applications of Optimization, Second Edition (Power Engineering), CRC Press, 2008.

[12] Fogel, D.B., Hays, T.J., Han, S.L. \& Quon, A Self-learning Evolutionary Chess Program,

Proc. IEEE, Vol. 92, No. 12, December, p.19471954, 2008 